\title{
A Magnetic Field-Independent Absorbing Boundary Condition for Magnetized Cold Plasma
}

\author{
Yaxin Yu, Student Member, IEEE, and Jamesina J. Simpson, Member, IEEE
}

\begin{abstract}
An effective absorbing boundary condition (ABC) based on the second-order approximations of Engquist and Majda's wave equations is presented for terminating three-dimensional finite-difference time-domain (FDTD) models employing the E-J collocated magnetized cold plasma algorithm. Numerical tests demonstrate that this $\mathrm{ABC}$ can effectively terminate magnetized plasma models involving arbitrary and spatially varying directions of the background magnetic field, a capability that is not provided by the recently developed KPML or NIMPML for magnetized cold plasma. This new capability will permit implementation of the Engquist and Majda-based $\mathrm{ABC}$ of this letter into localized and particularly global FDTD models of the Earth-ionosphere system involving varying directions of the magnetic field throughout the grid. An accuracy and stability analysis of this $\mathrm{ABC}$ is presented. Reflection errors are shown to be consistently less than $5 \%$ for various magnetic field directions and for magnetized plasmas having different electron densities by incorporating the phase velocity calculated at the center frequency of the source spectrum into the $\mathrm{ABC}$ formulation.
\end{abstract}

Index Terms-Absorbing boundary conditions (ABCs), electromagnetic propagation in plasma media, finite-difference methods, ionosphere, plasmas.

\section{INTRODUCTION}

$\mathbf{T}$ HE FULL-VECTOR Maxwell's equations finite-difference time-domain (FDTD) [1] method has been applied to modeling a variety of electromagnetic wave propagation phenomena in the Earth-ionosphere system [2], [3]. For example, local two-dimensional (2-D) as well as global three-dimensional (3-D) FDTD simulations have been generated to simulate propagation from lightning strikes, Schumann resonances, remote sensing of ionospheric disturbances, and remote sensing of oil fields [2], [3].

In 2006, a 2-D E-J collocated magnetized cold plasma FDTD algorithm [4] was developed for efficient modeling of electromagnetic wave propagation at higher frequencies and altitudes in the ionosphere than isotropic conductivity profiles previously permitted [2], [3]. This model was subsequently extended to 3-D in 2009 [5]. In order to model local regions of the Earth, or to model unbounded problems in the radial (vertical direction) on a global scale, the ionospheric FDTD plasma models of [4] and [5] require an appropriate absorbing boundary condition (ABC). Currently, the most efficient and commonly used $\mathrm{ABC}$ for the FDTD method is the perfectly matched layer (PML), initially

Manuscript received March 02, 2011; accepted March 28, 2011. Date of publication April 07, 2011; date of current version April 18, 2011. This work was supported by the National Science Foundation under CAREER Award Grant 0955404.

The authors are with the Department of Electrical and Computer Engineering, University of New Mexico, Albuquerque, NM 87131 USA (e-mail: yuy@unm. edu; simpson@ece.unm.edu).

Digital Object Identifier 10.1109/LAWP.2011.2139191 introduced by Berenger [6] in 1994. Its original split-field formulation and various later reformulations, such as the uniaxial PML (UPML) [7], the convolutional PML (CPML) [8], and the NPML [9], provide orders-of-magnitude lower reflections and other advantages [1] over previous versions of ABCs. However, the application of the standard PMLs has been limited to situations wherein the group and phase velocities of the incident wave are aligned in the same direction. When this is not the case, the traditional PML (as well as CPML, as confirmed by the authors) ceases to attenuate the field and can in fact act as an amplifier [10]-[12]. This is inherent in the PMLs and is not implementation-specific [12]. This poses a problem for modeling wave propagation in anisotropic media, such as whistler-mode waves in magnetized cold plasma.

To address this issue, Cummer [12] derives a modified PML solution for the negative index material (NIM) referred to as the NIMPML, which is an adaptation of the previous NPML [9]. However, this method requires the simulated material to have a homogeneous permittivity and permeability $\left(\varepsilon_{\mathrm{r}}=\mu_{\mathrm{r}}\right)$, which is hardly the case for the ionospheric plasma medium that varies with both altitude and spatial position around the Earth. More recently, Chevalier et al. [10] presented a new PML formulation in 2006 referred to as the KPML which explicitly utilizes information on the $\mathrm{k}$-vector direction of incident waves. Numerical tests in [10] demonstrate that KPML does not perform as well as the traditional CPML in open free-space problems, but it is stable and attenuates outgoing waves well for a case of whistler wave propagation in magnetized plasma media. However, the KPML can only be used as an effective $\mathrm{ABC}$ for dense magnetized plasma when the electron cyclotron frequency is much smaller than the plasma frequency. This is not the case for the lower region of ionosphere. Moreover, the KPML algorithm is based on a single orientation of the background magnetic field relative to each side of the FDTD grid and, as such, is not suitable for simulations involving spatially varying magnetic field directions such as for global modeling of the Earth-ionosphere system.

Considering these issues relating to the application of PMLs to magnetized plasma, we consider Mur's ABC [13] as an alternative. Unfortunately, it is known that the original Mur's $\mathrm{ABC}$ can exhibit an early-time instability for certain modeling scenarios as discussed in [14] and [15], and this includes magnetized plasma problems as we have found through numerical tests. To solve this issue, Zhang and Wang [14] present a different discretization scheme of the Engquist and Majda's second-order $\mathrm{ABC}$ equations [16], which they claim is stable. However, we find through longer numerical tests that the early-time instability of [13] is simply replaced by a late-time instability in [14]. 
In this letter, we investigate the feasibility of a variation of the Zhang and Wang's scheme [14] to serve as an effective ABC for the 3-D FDTD magnetized cold plasma formulation of [5]. We choose to start with this scheme despite its inherent late-time instability because it has the potential to provide an acceptable level of error regardless of the direction of the background magnetic field, a capability not provided by KPML. We then present a simple but very effective way to further delay the late-time instability of this magnetized plasma ABC.

\section{FORMULATION}

The Engquist and Majda's second-order ABC equation [16] along the outer $x=1$ boundary of a 3-D Cartesian FDTD lattice can be expressed as

$$
\frac{\partial^{2} U}{\partial t \partial x}-\frac{1}{v} \frac{\partial^{2} U}{\partial t^{2}}+\frac{v}{2} \frac{\partial^{2} U}{\partial y^{2}}+\frac{v}{2} \frac{\partial^{2} U}{\partial z^{2}}=0
$$

One can also easily obtain the expressions for other outer boundaries. $U$ denotes the scalar field components located at the outer boundary, and $v$ is the wave phase velocity, which is initially set to $c$, the velocity in free space. By central- differentiating the partial and second-order time and spatial derivatives of (1) at time-step $n$ and at the spatial locations one-half grid cell inside the $x=1$ boundary, one can obtain the well-known second-order Mur's ABC. In this letter, by utilizing a different discretization scheme of (1) than that utilized in [14], we derive the $\mathrm{ABC}$ expression of [14] as detailed below (that is, a different approach from the so-called upwind-difference scheme of [14] is utilized, but we arrive at the same final form of the equation).

First, $U$ around time-step $n$ and spatial location $(i+1, j, k)$ can be Taylor-expanded as $\left.U\right|_{\mathrm{n},(\mathrm{i}+1, j, k)}=\left.U\right|_{\mathrm{n},(\mathrm{i}, j, k)}+$ $\left[\left.(\partial U / \partial x)\right|_{\mathrm{n},(\mathrm{i}, j, k)}\right] \cdot \Delta x+O\left[\Delta x^{2}\right]$. The first-order finite-difference approximation of the space derivative can then be obtained as $\left.(\partial U / \partial x)\right|_{\mathrm{n},(\mathrm{i}, j, k)}=\left[\left.U\right|_{\mathrm{n},(\mathrm{i}+1, j, k)}-\left.U\right|_{\mathrm{n},(\mathrm{i}, j, k)}\right] / \Delta x$. Applying this approximation to both the spatial and time derivatives for the first term of (1), we obtain the following:

$$
\begin{aligned}
\left.\frac{\partial^{2} U}{\partial x \partial t}\right|_{1, j, k} ^{n} & =\frac{1}{\Delta t}\left[\left.\frac{\partial U}{\partial x}\right|_{1, j, k} ^{n}-\left.\frac{\partial U}{\partial x}\right|_{1, j, k} ^{n-1}\right] \\
& =\frac{1}{\Delta t} \cdot\left[\frac{\left.U\right|_{2, j, k} ^{n}-\left.U\right|_{1, j, k} ^{n}}{\Delta x}-\frac{\left.U\right|_{2, j, k} ^{n-1}-\left.U\right|_{1, j, k} ^{n-1}}{\Delta x}\right] \\
& =\frac{\left.U\right|_{1, j, k} ^{n-1}-\left.U\right|_{2, j, k} ^{n-1}}{\Delta t \Delta x}-\frac{\left.U\right|_{1, j, k} ^{n}-\left.U\right|_{2, j, k} ^{n}}{\Delta t \Delta x}
\end{aligned}
$$

Next, the central-difference scheme is applied to the last three second-order time and spatial derivatives of (1) at time-step $n$ and the $x=1$ plane. Equation (1) can now be expressed as

$$
\begin{aligned}
\frac{\left.U\right|_{1, j, k} ^{n-1}-\left.U\right|_{2, j, k} ^{n-1}}{\Delta t \Delta x} & -\frac{\left.U\right|_{1, j, k} ^{n}-\left.U\right|_{2, j, k} ^{n}}{\Delta t \Delta x} \\
& -\frac{\left.U\right|_{1, j, k} ^{n+1}-\left.2 U\right|_{1, j, k} ^{n}+\left.U\right|_{1, j, k} ^{n-1}}{v \cdot \Delta t^{2}} \\
& +\frac{v\left(\left.U\right|_{1, j+1, k} ^{n}-\left.2 U\right|_{1, j, k} ^{n}+\left.U\right|_{1, j-1, k} ^{n}\right)}{2 \cdot \Delta y^{2}} \\
& +\frac{v\left(\left.U\right|_{1, j, k+1} ^{n}-\left.2 U\right|_{1, j, k} ^{n}+\left.U\right|_{1, j, k-1} ^{n}\right)}{2 \cdot \Delta z^{2}}=0 .
\end{aligned}
$$

By moving the field components of time-step $n$ and $n-1$ to the right-hand side, (3) is rewritten as

$$
\begin{aligned}
\left.U\right|_{1, j, k} ^{n+1}= & \left.2 U\right|_{1, j, k} ^{n}-\left.U\right|_{1, j, k} ^{n-1}-\frac{v \Delta t}{\Delta x}\left[\left.U\right|_{1, j, k} ^{n}-\left.U\right|_{2, j, k} ^{n}\right] \\
& +\frac{v \Delta t}{\Delta x}\left[\left.U\right|_{1, j, k} ^{n-1}-\left.U\right|_{2, j, k} ^{n-1}\right] \\
& +\frac{1}{2}\left(\frac{v \Delta t}{\Delta y}\right)^{2} \cdot\left[\left.U\right|_{1, j+1, k} ^{n}-\left.2 U\right|_{1, j, k} ^{n}+\left.U\right|_{1, j-1, k} ^{n}\right] \\
& +\frac{1}{2}\left(\frac{v \Delta t}{\Delta z}\right)^{2} \cdot\left[\left.U\right|_{1, j, k+1} ^{n}-\left.2 U\right|_{1, j, k} ^{n}+\left.U\right|_{1, j, k-1} ^{n}\right] .
\end{aligned}
$$

As mentioned earlier, this $\mathrm{ABC}$ formulation exhibits a latetime instability that is improved over the early-time instability of the traditional Mur's ABC for magnetized plasma. Thus, to further delay the start of this late-time instability, we intentionally multiply the newly updated field components along the $\mathrm{ABC}$ boundaries each time-step by a stability control factor $s$ slightly less than 1.0. Then, (4) is re-expressed as

$$
\begin{aligned}
\left.U\right|_{1, j, k} ^{n+1}= & s \cdot\left\{\left.2 U\right|_{1, j, k} ^{n}-\left.U\right|_{1, j, k} ^{n-1}-\frac{v \Delta t}{\Delta x}\left[\left.U\right|_{1, j, k} ^{n}-\left.U\right|_{2, j, k} ^{n}\right]\right. \\
& +\frac{v \Delta t}{\Delta x}\left[\left.U\right|_{1, j, k} ^{n-1}-\left.U\right|_{2, j, k} ^{n-1}\right]+\frac{1}{2}\left(\frac{v \Delta t}{\Delta y}\right)^{2} \\
& \cdot\left[\left.U\right|_{1, j+1, k} ^{n}-\left.2 U\right|_{1, j, k} ^{n}+\left.U\right|_{1, j-1, k} ^{n}\right]+\frac{1}{2}\left(\frac{v \Delta t}{\Delta z}\right)^{2} \\
& \left.\cdot\left[\left.U\right|_{1, j, k+1} ^{n}-\left.2 U\right|_{1, j, k} ^{n}+\left.U\right|_{1, j, k-1} ^{n}\right]\right\}
\end{aligned}
$$

wherein $s=1.0$ yields the original ABC (4). It is expected that this multiplication factor will increase the level of reflection from the boundary, but it will further improve the stability (in the extreme case, $s=0.0$ yields a PEC boundary wherein all the wave are fully reflected, but the algorithm is completely stable).

Equation (5) is the final ABC expression for the $x=1$ boundary. It is used to terminate the magnetized cold plasma in the numerical studies of Section III. The ABC formulations for all other outer boundaries can also be derived similarly.

\section{NumERICAL RESULTS}

The performance of our $\mathrm{ABC}$ scheme is first evaluated by testing the reflection error in a $100 \times 100 \times 100$-cell FDTD grid having an $x$-directed electric dipole source at its center with a Gaussian-modulated sinusoidal waveform $E_{\mathrm{x}}=\exp [-[(t-$ $\left.\left.\left.t_{0}\right) / t_{w}\right]^{2}\right] \cdot \sin \left(2 \pi f_{c} \cdot t\right)$, where $t_{w}=2.93 \mu \mathrm{s}, t_{0}=2.2 t_{w}$, and $f_{c}=4.0 \mathrm{MHz}$ is the center frequency of the source. The lattice space increments in each Cartesian direction of the FDTD grid are $d x=d y=d z=5 \mathrm{~m}$, the time-step $d t$ is 0.97 times the Courant limit [1], and the stability control factor is $s=1$. The plasma medium has an electron density $N_{\mathrm{e}}$ of $1.86 \times 10^{8} \mathrm{~m}^{-3}$, which corresponds to the ionosphere at an altitude of about $90 \mathrm{~km}$. A static background magnetic field of $1 \times 10^{-5} \mathrm{~T}$ of various orientations is applied to the plasma to approximately mimic the magnetic field of the Earth. Without loss of generality, the $E_{\mathrm{x}}$ field component is probed at a point in the same $x y$ plane as the source and three cells from the outer $y$-boundary. The relative reflection error Error $[n,(i, j, k)]$ of the $\mathrm{ABC}$ at 


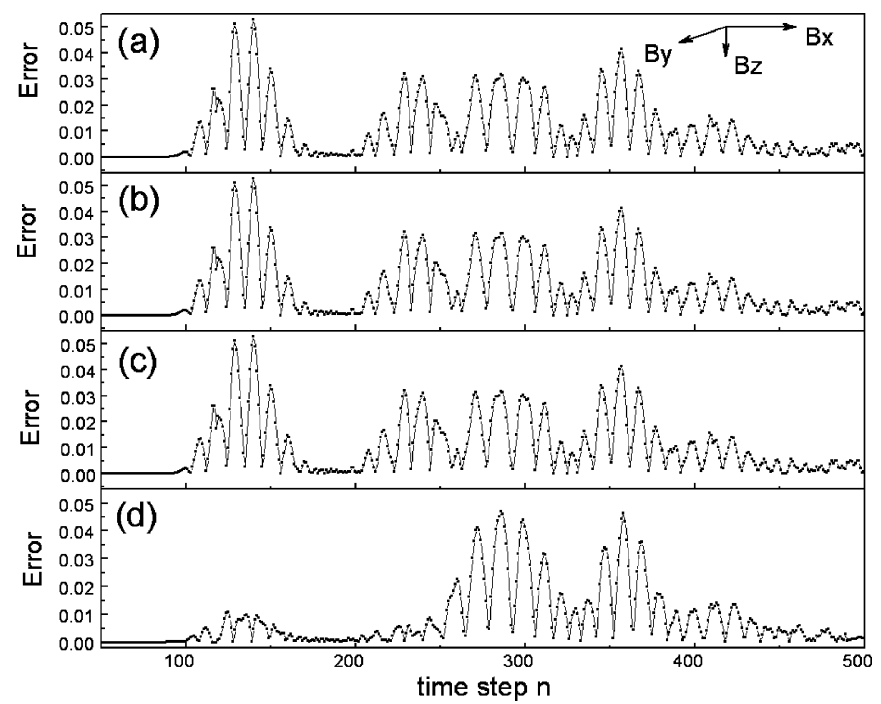

Fig. 1. Reflection errors for different magnetic field directions applied to the plasma. (a) $B_{\mathrm{x}}=B_{\mathrm{y}}=0 \mathrm{~T}, B_{\mathrm{z}}=-1 \times 10^{-5} \mathrm{~T}$. (b) $B_{\mathrm{x}}=1 \times 10^{-5} \mathrm{~T}$, $B_{\mathrm{y}}=B_{\mathrm{z}}=0 \mathrm{~T}$ for $z \geq z_{\max } / 2$, and $B_{\mathrm{z}}=-1 \times 10^{-5} \mathrm{~T}, B_{\mathrm{x}}=B_{\mathrm{y}}=0 \mathrm{~T}$ for $z<z_{\max } / 2$. (c) $B_{\mathrm{x}}=B_{\mathrm{y}}=0.5 \times 10^{-5} \mathrm{~T}, B_{\mathrm{z}}=-0.707 \times 10^{-5} \mathrm{~T}$. (d) Same magnetic field as in (c) applied to Mur's ABC.

time-step $n$ and grid location $(i, j, k)$ is then calculated by the method detailed in [1] and defined as

$$
\operatorname{Error}[n,(i, j, k)]=\frac{\left|E[n,(i, j, k)]-E_{\text {ref }}[n,(i, j, k)]\right|}{\left|E_{\text {ref.max }}[(i, j, k)]\right|}
$$

where $E[n,(i, j, k)]$ is the electric field value recorded at the probing point, and the reference solution $E_{\mathrm{ref}}[n,(i, j, k)]$ is obtained from a sufficiently large benchmark grid having no reflected signals from the grid boundaries at the probing point over the time-stepping span of interest. The maximum amplitude of the reference fields over all time-steps at grid location $(i, j, k)$ is denoted as $E_{\text {ref.max }}[(i, j, k)]$.

Fig. 1(a)-(c) illustrates the numerical results of the relative errors for different applied magnetic field directions. In Fig. 1(a), a $z$-directed magnetic field is applied to the plasma $\left(B_{\mathrm{z}}=\right.$ $-1 \times 10^{-5} \mathrm{~T}$ and $B_{\mathrm{x}}=B_{\mathrm{y}}=0 \mathrm{~T}$ ). In Fig. 1(b), an $x$-directed magnetic field is applied to the plasma in the upper half of the grid $\left(z \geq z_{\max } / 2, B_{\mathrm{x}}=1 \times 10^{-5} \mathrm{~T}\right.$ and $\left.B_{\mathrm{y}}=B_{\mathrm{z}}=0 \mathrm{~T}\right)$ and a $z$-directed magnetic field is applied to the plasma in the lower half of the grid $\left(z<z_{\max } / 2, B_{\mathrm{z}}=-1 \times 10^{-5} \mathrm{~T}\right.$ and $B_{\mathrm{x}}=B_{\mathrm{y}}=0 \mathrm{~T}$ ), where $z_{\max }$ is the maximum number of grid cells in the $z$-direction. In Fig. 1(c), a magnetic field with $45^{\circ}$ codip and Azimuth angles is applied to the plasma $\left(B_{\mathrm{x}}=B_{\mathrm{y}}=0.5 \times 10^{-5} \mathrm{~T}\right.$ and $\left.B_{\mathrm{z}}=-0.707 \times 10^{-5} \mathrm{~T}\right)$. For all the cases depicted in Fig. 1(a)-(c), the reflection errors are seen to be less than $\sim 5 \%$, clearly demonstrating the ABC's capability to provide the same approximate level of error regardless of the applied magnetic field direction.

Next, to evaluate the newly derived ABC's performance compared to the original Mur's ABC, the same simulation parameters of Fig. 1(c) are repeated with the original Mur's ABC. Fig. 1(d) shows that the maximum reflection error for the case of the original Mur's $\mathrm{ABC}$ is also around 5\%, as for the $\mathrm{ABC}$ of this letter.

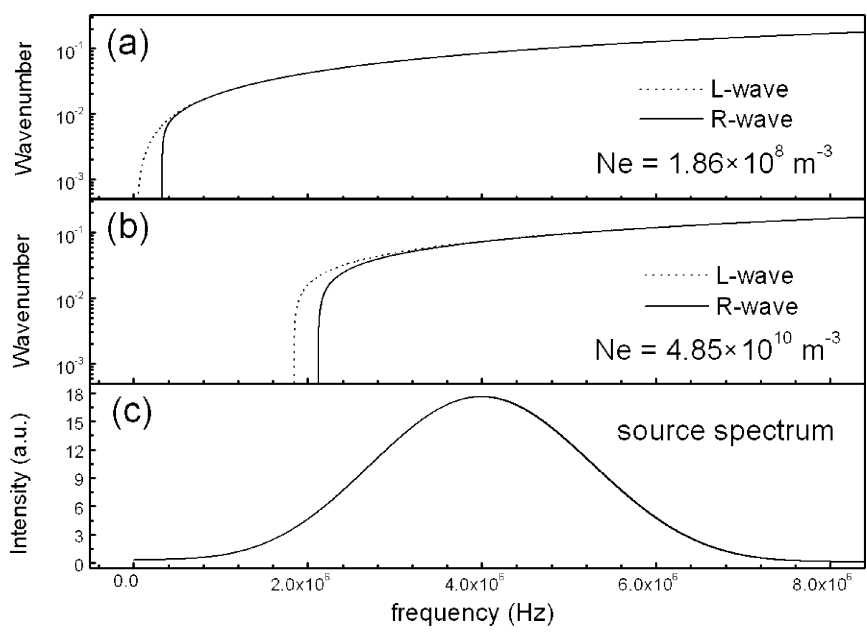

Fig. 2. (a) Dispersion diagram for a low-density plasma $\left(N_{\mathrm{e}}=1.86 \times\right.$ $\left.10^{8} \mathrm{~m}^{-3}\right)$. (b) Dispersion diagram for a high-density plasma $\left(N_{\mathrm{e}}=\right.$ $\left.4.85 \times 10^{10} \mathrm{~m}^{-3}\right)$. (c) Source wave spectrum.

In Fig. 1, we performed accuracy studies of the $\mathrm{ABC}$ at a relatively low altitude $(\sim 90 \mathrm{~km})$ of the ionosphere (therefore a relatively low-density plasma). To study the ABC's performance at higher altitudes (more dense plasmas), the same simulation of Fig. 1(a) is repeated for progressively larger electron densities $N_{\mathrm{e}}$. We find through a series of tests that the maximum reflection error remains at the same level in Fig. 1(a) until the electron density $N_{\mathrm{e}}$ is increased to a level such that the center frequency $f_{\mathrm{c}}$ of the source becomes near to the critical cutoff frequencies of the plasma. For example, Fig. 2(c) illustrates the source spectrum employed in all of the numerical experiments of this letter. Fig. 2(a) shows the dispersion diagram (wavenumber versus wave frequency) for the case of Fig. 1(a) (a low-density plasma with $N_{\mathrm{e}}=1.86 \times 10^{8} \mathrm{~m}^{-3}$ corresponding to an altitude of $\sim 90 \mathrm{~km}$ ), and Fig. 2(b) shows the dispersion diagram for a higher-density plasma with $N_{\mathrm{e}}=$ $4.85 \times 10^{10} \mathrm{~m}^{-3}$ (corresponding to an altitude of $\sim 400 \mathrm{~km}$ ).

The dotted lines of Fig. 2(a) and (b) represent the dispersion relation of the left-hand circular polarization wave (L-wave), and the solid lines represent the dispersion diagram of the righthand circular wave (R-wave). We note that according to Fig. 2, as $N_{\mathrm{e}}$ is increased from $1.86 \times 10^{8} \mathrm{~m}^{-3}$ to $4.85 \times 10^{10} \mathrm{~m}^{-3}$, the source begins to have substantial spectrum below the cutoff frequencies of the L-and R-waves. This causes the portion of the source spectrum below $\sim 2.1 \mathrm{MHz}$ to change from propagating modes as in the case of Fig. 2(a) to nonpropagating modes as in the case of Fig. 2(b). More importantly, though, closer to the cutoff frequencies, the phase velocity is increased. As a result, because a velocity of $c$ corresponding to free space has been assumed for the $\mathrm{ABC}$, the reflection error increases from $\sim 5 \%$ as in Fig. 1(a) to $\sim 6 \%$ as shown in Fig. 3(a) for the same scenario except for having increased $N_{\mathrm{e}}$. To improve the accuracy, instead of using the free-space speed $c$ for $v$ in equation (6), the phase velocity $v$ in the plasma at the source center frequency $f_{\mathrm{c}}$ is utilized, resulting in a value of $\sim 1.13 c$. Fig. 3(b) presents the results using $v=1.13 c$, and as expected, the maximum reflection error is now reduced to $\sim 4.5 \%$ from the $\sim 6 \%$ of Fig. 3(a). 


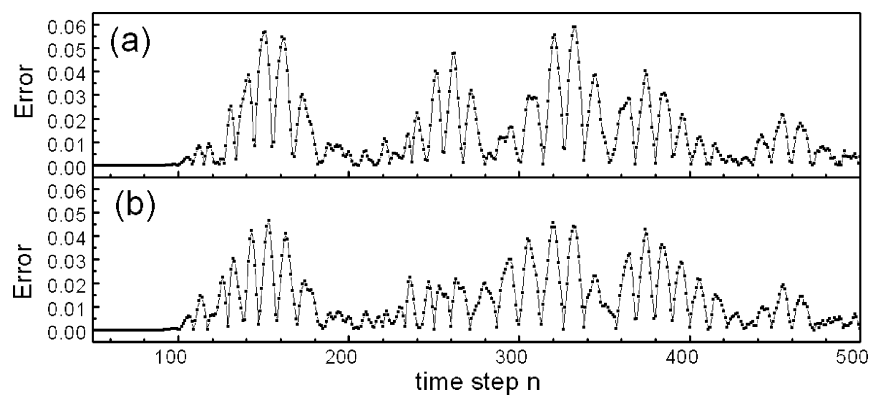

Fig. 3. Reflection errors for a dense plasma $\left(N_{\mathrm{e}}=4.85 \times 10^{10} \mathrm{~m}^{-3}\right)$. (a) Phase velocity $v$ of the ABC assumed as free-space speed $c$. (b) $v=1.13 \mathrm{c}$.

Finally, numerical simulations have been conducted to test the ability to further delay the start of the late-time instability using the control factor $s$. For these tests, the plasma and magnetic field parameters are the same as for Fig. 1(a). For the case of no stability control $(s=1.0)$, the instability starts around $\sim 5000$ time-steps. For the case of stability control $(s=0.94)$, the algorithm remains stable through $~ 9000$ time-steps. As a comparison, the early-time instability introduced by the original Mur's ABC occurs at 1000 time-steps.

As mentioned earlier, we expect that applying a stability control factor will increase the reflection error. In fact, the original $\sim 5 \%$ error provided by the $s=1.0$ case of Fig. 1(a) is increased here to $\sim 6 \%$ for the $s=0.94$ case. On the other hand, the simulation is now stable for nearly double the number of time-steps. Thus, to use the control factor effectively in general modeling scenarios, a value for $s$ should be carefully chosen on a case-by-case basis to provide the proper balance between controlling the instability and the need for better accuracy.

\section{CONCLUSION AND ONGOING WORK}

We have discretized the Engquist and Majda's second-order wave equations using a variation of the approach utilized by Zhang and Wang [14] to serve as an ABC for FDTD grids employing the 3-D E-J collocated magnetized cold plasma algorithm [5]. We have demonstrated that reflection errors can be maintained to be less than $5 \%$ along the boundaries by utilizing the phase velocity in the magnetized plasma calculated at the center frequency of the source spectrum in the $\mathrm{ABC}$ formulations instead of the original free-space velocity $c$ preassumed in Engquist and Majda's second-order wave (1). Moreover, we have introduced a technique to further delay the late-time instability of the algorithm of [14] by a factor of about two. A very important advantage of this method over the KPML is that it performs well for spatially varying magnetic field directions of a magnetized plasma. This is a very important characteristic for ionosphere modeling, particularly for global modeling of electromagnetic wave propagation that includes varying directions of the geomagnetic field throughout the grid, and includes a wide range of plasma parameters at different positions and altitudes.
Ongoing work includes applying the ABC developed here to the upper radial direction of the latitude-longitude [17] and geodesic [18] global FDTD models of the Earth-ionosphere system. The 3-D ABC of this letter may also be utilized to generate localized 3-D Earth-ionosphere system models.

\section{REFERENCES}

[1] A. Taflove and S. C. Hagness, Computational Electromagnetics: Finite-Difference Time-Domain Method, 3rd ed. Norwood, MA: Artech House, 2005.

[2] J. J. Simpson and A. Taflove, "A review of progress in FDTD Maxwell's equations modeling of impulsive sub-ionospheric propagation below $300 \mathrm{kHz}$," IEEE Trans. Antennas Propag., vol. 55, no. 6, pp. 1582-1590, Jun. 2007.

[3] J. J. Simpson, "Current and future applications of 3-D global Earthionosphere models based on the full-vector Maxwell's equations FDTD method," Surveys Geophys., vol. 30, no. 2, pp. 105-130, 2009.

[4] W. Hu and S. A. Cummer, "An FDTD model for low and high altitude lightning-generated EM fields," IEEE Trans. Antennas Propag., vol. 54, no. 5, pp. 1513-1522, May 2006.

[5] Y. Yu and J. J. Simpson, "An E-J collocated 3-D FDTD model of electromagnetic wave propagation in magnetized cold plasma," IEEE Trans. Antennas Propag., vol. 58, no. 2, pp. 469-478, Feb. 2010.

[6] J. P. Berenger, "A perfectly matched layer for the absorption of electromagnetic waves," J. Comput. Phys., vol. 114, pp. 185-200, 1994.

[7] S. G. Gedney, "An anisotropic perfectly matched layer-absorbing medium for the truncation of FDTD lattices," IEEE Trans. Antennas Propag., vol. 44, no. 12, pp. 1630-1639, Dec. 1996.

[8] J. A. Roden and S. D. Gedney, "Convolutional PML (CPML): An efficient FDTD implementation of the CFS-PML for arbitrary media," Microw. Opt. Technol. Lett., vol. 27, no. 5, pp. 334-339, 2000.

[9] S. A. Cummer, "A simple, nearly perfectly matched layer for general electromagnetic media," IEEE Microw. Wireless Compon. Lett., vol. 13, no. 3, pp. 128-130, Mar. 2003.

[10] M. W. Chevalier, T. W. Chevalier, and U. S. Inan, "A PML utilizing $\mathrm{k}$-vector information as applied to the whistler mode in a magnetized plasma," IEEE Trans. Antennas Propag., vol. 54, no. 8, pp. 2424-2429, Aug. 2006.

[11] E. Becache, S. Fauqueux, and P. Joly, "Stability of perfectly matched layers, group velocities, and anisotropic waves," J. Comput. Phys., vol. 188 , pp. 399-433, 2003.

[12] S. A. Cummer, "Perfectly matched layer behavior in negative refractive index materials," IEEE Antennas Wireless Propag. Lett., vol. 3, pp. 172-175, 2004

[13] G. Mur, "Absorbing boundary conditions for the finite-difference approximation of time-domain electromagnetic field equations," IEEE Trans. Electromagn. Compat., vol. EMC-23, no. 4, pp. 377-382, Nov. 1981.

[14] Z. Yusheng and W. Wenbing, "The studies of the stability of FDTD with Mur's absorbing boundary condition of second order in 3-D scattering problems," IEEE Microw. Guided Wave Lett., vol. 6, no. 3, pp. 120-122, Mar. 1996.

[15] J.-N. Hwang and F.-C. Chen, "Stability analysis of the Mur's absorbing boundary condition in the alternating direction implicit finite-difference method," Microw. Antennas Propag., vol. 1, no. 3, pp. 597-601, Jun. 2007.

[16] B. Engquist and A. Majda, "Absorbing boundary conditions for the numerical simulation of waves," Math. Comput., vol. 31, pp. 629-651, 1977.

[17] J. J. Simpson and A. Taflove, "Three-dimensional FDTD modeling of impulsive ELF antipodal propagation and Schumann resonance of the Earth-sphere," IEEE Trans. Antennas Propag., vol. 52, no. 2, pp. 443-451, Feb. 2004

[18] J. J. Simpson, R. P. Heikes, and A. Taflove, "FDTD modeling of a novel ELF radar for major oil deposits using a three-dimensional geodesic grid of the Earth-ionosphere waveguide," IEEE Trans. Antennas Propag., vol. 54, no. 6, pp. 1734-1741, Jun. 2006. 